\title{
Effects of Capping of the Tracheostomy Tube in Stroke Patients With Dysphagia
}

\author{
Yong kyun Kim, MD, PhD, Sang-heon Lee, MD, Jang-won Lee, MD
}

Department of Physical Medicine and Rehabilitation, Myongji Hospital, Seonam University College of Medicine, Goyang, Korea

Objective To investigate the impact of tracheostomy tube capping on swallowing physiology in stroke patients with dysphagia via videofluoroscopic swallowing study (VFSS).

Methods This study was conducted as a prospective study that involved 30 stroke patients. Then, $4 \mathrm{~mL}$ semisolid swallowing was conducted with capping of the tracheostomy tube or without capping of the tracheostomy tube. The following five parameters were measured: laryngeal elevation, pharyngeal transit time, post-swallow pharyngeal remnant, upper esophageal sphincter width (UES), and penetration-aspiration scale (PAS) score.

Results On assessment of the differences in swallowing parameters during swallowing between 'with capping' and 'without capping' statuses, statistically significant differences were found in the post-swallow pharyngeal remnant (without capping, $48.19 \% \pm 28.70 \%$; with capping, $25.09 \% \pm 19.23 \%$; $\mathrm{p}<0.001$ ), normalized residue ratio scale for the valleculae (without capping, $0.17 \pm 0.12$; with capping, $0.09 \pm 0.12$; $\mathrm{p}=0.013$ ), normalized residue ratio scale for the piriform sinus (without capping, $0.16 \pm 0.12$; with capping, $0.10 \pm 0.07 ; \mathrm{p}=0.015$ ), and UES width (without capping, $3.32 \pm 1.61 \mathrm{~mm}$; with capping, $4.61 \pm 1.95 \mathrm{~mm}$; $\mathrm{p}=0.003)$. However, there were no statistically significant differences in laryngeal elevation ( $\mathrm{x}$-axis without capping, $2.48 \pm 1.45 \mathrm{~mm}$; with capping, $3.26 \pm 2.37 \mathrm{~mm}$; $\mathrm{y}$-axis without capping, $11.11 \pm 5.24 \mathrm{~mm}$; with capping, $12.64 \pm 6.16 \mathrm{~mm}$ ), pharyngeal transit time (without capping, 9.19 \pm $10.14 \mathrm{~s}$; with capping, $9.09 \pm 10.21 \mathrm{~s}$ ), and PAS score (without capping, 4.94 \pm 2.83 ; with capping, 4.18 \pm 2.24 ).

Conclusion Tracheostomy tube capping is a useful way to reduce post-swallow remnants and it can be considered an alternative method for alleviating dysphagia in stroke patients who can tolerate tracheostomy tube capping when post-swallow remnants are observed.

Keywords Tracheostomy, Stroke, Deglutition disorders, Fluoroscopy

\section{INTRODUCTION}

Tracheostomy is a critical-care patient management procedure required during emergency conditions such as acute or chronic respiratory failure involving upper airway obstruction and the need for mechanical ventilation,

Received May 16, 2016; Accepted September 9, 2016

Corresponding author: Jang-won Lee

Department of Physical Medicine and Rehabilitation, Myongji Hospital, Seonam University College of Medicine, 55 Hwasu-ro 14beon-gil, Deokyanggu, Goyang 10475, Korea. Tel: +82-31-810-6450, Fax: +82-31-810-6563, E-mail: rallo1080@naver.com

ORCID: Yong kyun Kim (http://orcid.org/0000-0002-1224-8965); Sang-heon Lee (http://orcid.org/0000-0003-2770-8562); Jang-won Lee (http://orcid. org/0000-0002-2091-6150).

(c) This is an open-access article distributed under the terms of the Creative Commons Attribution Non-Commercial License (http://creativecommons.org/ licenses/by-nc/4.0) which permits unrestricted noncommercial use, distribution, and reproduction in any medium, provided the original work is properly cited. Copyright (c) 2017 by Korean Academy of Rehabilitation Medicine 
airway clearance, etc. It may cause various complications, ranging from acute complications like hemorrhage, infection, and tracheal obstruction to chronic complications like tracheal stenosis, tracheocutaneous fistula, and tracheoesophageal fistula $[1,2]$.

Dysphagia subsequent to stroke is a common problem $[3,4]$ that causes aspiration pneumonia and may increase the morbidity and mortality risk [5]. Tracheostomy is frequently performed depending on the severity of the stroke [6-8], but tracheostomy itself may exacerbate dysphagia $[9,10]$. The presence of a tracheostomy tube causes reduced upward and anterior movement of the larynx [11], decreased cough effectiveness due to lowered tracheal sensitivity, and physiologic changes such as relative esophageal obstruction due to the tracheostomy tube cuff itself, and the glottic closure reflex declines due to decreased proprioception [12]. Moreover, the vocal cord closure time reduces, and if vocal cord palsy is present, which may accompany a stroke, the incidence of aspiration may increase [13]. Alteration of the subglottic pressure also occurs, which is associated with dysphagia. During normal swallowing, positive subglottic pressure is created [14], and with an open tracheostomy tube, glottic airflow leaks and loss of subglottic pressure occur. Therefore, many researchers have made attempts to preserve the subglottic pressure, such as inserting a one-way speaking valve, capping of the tracheostomy tube, and decannulation [15-18].

In a previous study that applied a Passy-Muir valve [19], the measured peak pressure during swallowing was increased up to $10 \mathrm{~cm} \mathrm{H}_{2} \mathrm{O}$. During exhalation, positive subglottic air pressure was created [19]. Improvement in swallowing efficiency and reduction in aspiration were also noted [19]. However in that study, only the presence of aspiration was assessed, and the outcome measurements that could be confirmed on videofluoroscopic swallowing study (VFSS), such as the post-swallow remnant, pharyngeal transit time, laryngeal elevation, and upper esophageal sphincter (UES) width, were not measured. Kim et al. [15] investigated the alleviation of dysphagia through the reduced anchoring effect of the tracheostomy tube and preservation of the subglottic air pressure after decannulation. Improvement in aspiration was not observed, but improvement in the post-swallow remnant and UES width was reported.

Previous studies on preservation of the subglottic pres- sure showed different outcomes. In this study, tracheostomy tube capping was performed in stroke patients. The outcome measurements were confirmed via VFSS to verify the hypothesis that preservation of the deglutitive subglottic pressure would effectively alleviate dysphagia.

\section{MATERIALS AND METHODS}

The Ethics Committee of Myongji Hospital approved this study. The Institutional Review Board (IRB) approval number is MJH-15-025. In this study, a data collector (author 1), a practitioner (author 2), and a data analyst (author 3) were involved. For reducing potential bias, all authors were blinded to each task.

\section{Subjects}

This study was conducted as a prospective study that involved 30 stroke patients who were hospitalized at the rehabilitation medicine department from May 2, 2015 to March 28, 2016. All patients underwent otorhinolaryngology consultation. Changing the tracheostomy tube (uncuffed type) for capping and evaluating the suitability of capping of the tracheostomy tube using laryngoscopy (the ENF-GP portable fiberoptic rhinolaryngoscope provided by Olympus Medical Systems Corp., Tokyo, Japan) were conducted.

Laryngoscopy was used to assess airway obstruction and vocal cord paralysis. Patients who did not have airway obstruction or vocal cord paralysis were considered appropriate for this study. The inclusion criteria were as follows: (1) patients with stroke caused by cerebral hemorrhage and/or infarction who received tracheostomy; and (2) patients who could tolerate capping of the tracheostomy tube during VFSS. Capping of the tracheostomy tube was performed by following the tracheal tube occlusion protocol described in the previously published paper presented by Rumbak et al. [20]. Patient's $\mathrm{O}_{2}$ saturation, blood pressure, heart rate, and respiratory rate were measured, and patients who had more than $90 \% \mathrm{O}_{2}$ saturation and who did not show a more than $15 \%$ change in the other parameters compared to the baseline values were included. On the other hand, the exclusion criteria were as follows: (1) patients with a structural abnormality in the oropharynx; and (2) patients with a structural abnormality in the cervical spine (e.g., patients with bony abnormalities such as an osteophyte, etc.). 


\section{Procedure}

VFSS was performed with a 4-mL bolus of semisolid paste in the chin-tuck position. Semisolid paste was prepared as a 2:1 volumetric mixture of dysphagia formula level 1 (foods should be pudding-like without lumps, chunks, or pulp) and contrast barium solution (SolotopHD provided by Taejoon Pharm Co. Ltd., Yongin, Korea). For the contrast solution, $375 \mathrm{~g}$ barium sulfate powder was mixed with $90 \mathrm{~mL}$ water. In this study, a semisolid, whose remnant amount could be calculated, was used. The patients with cognitive decline or who had difficulty maintaining head control were made to sit in a wheelchair, and an assistant held their head to help them maintain the said position. The images of the patients with a tracheostomy tube while swallowing a 4-mL bolus of semisolid paste once before and after capping were compared. Whether the first swallow would be performed before corking or after corking was determined by a coin toss. The practitioner conducted VFSS without having the patient's information.

\section{Measures}

The measurement method was similar to that used in Kim's previous study [15]. Measurement was performed twice based on the images of swallowing with capping and without capping. The following five parameters were measured: laryngeal elevation, pharyngeal transit time, post-swallow pharyngeal remnant, UES width, and aspiration or PAS scale score.

For assessing laryngeal elevation, a straight line was drawn for the y-axis from the anterior-inferior margin of the 4 th vertebral body to the anterior-inferior margin of the 2nd vertebral body, and the $\mathrm{x}$-axis was determined to be perpendicular to the $\mathrm{Y}$ axis. The difference $(\mathrm{mm})$ in the location of the hyoid bone before and after swallowing was obtained (Fig. 1). For assessing the pharyngeal transit time, the time interval(s) from the time point at which food passes the posterior nasal spine to the time point at which the food completely passes the pharyngoesophageal sphincter was measured. For assessing the post-swallow pharyngeal remnant, two measuring meth-
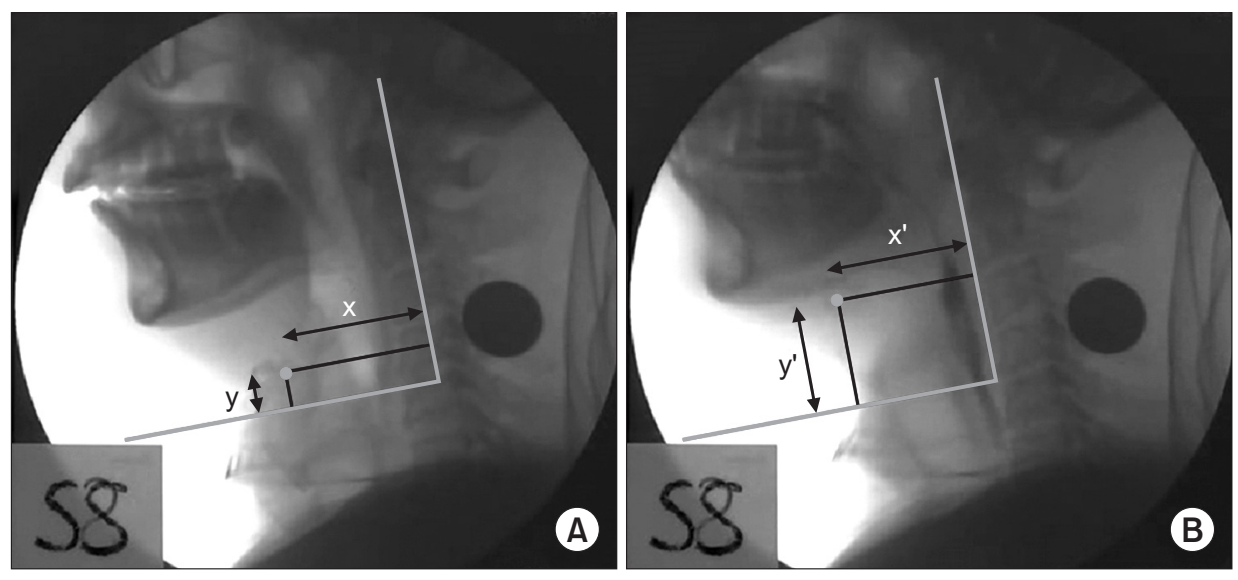

Fig. 1. Laryngeal elevation: after setting the anterior-inferior margin of the 4 th cervical vertebral body to 0 , the $y$-axis was based on the vertical connecting line of the anterior-inferior margin of the 2nd cervical vertebral body, and the $\mathrm{x}$-axis was defined as a line perpendicular to the $y$-axis. (A) Location of the hyoid bone before swallowing and (B) during swallowing, and the maximally elevated point of the hyoid bone.
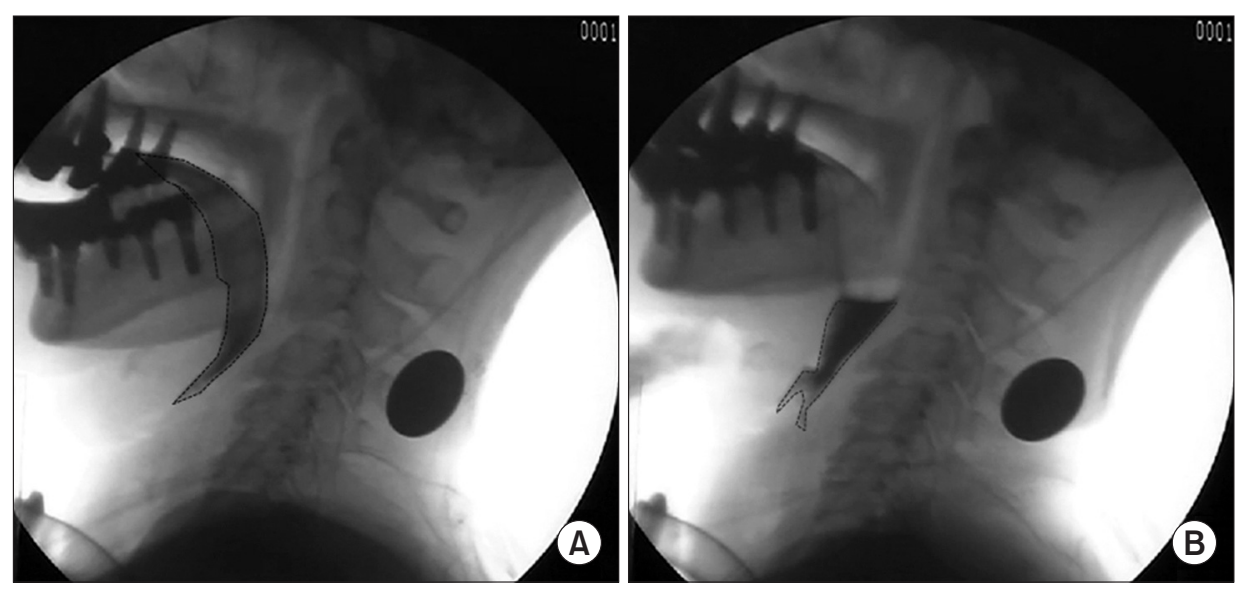

Fig. 2. Post-swallow pharyngeal remnant: difference in the remnant amount (\%) before and after swallowing measured with an AutoCAD 2D screen. (A) Remnant before swallowing. (B) Remnant after swallowing. 
ods were used. The first method measured the difference in the remnant amount (\%) before and after swallowing (Fig. 2). The second method measured the normalized residue ratio scale (NRRS), proposed by Pearson. It was used for evaluation of the piriform sinus as well as the post-swallow remnant of the valleculae [21]. For assessing the UES width, the length ( $\mathrm{mm}$ ) of the maximal opening of the narrowest region of C3-6 during swallowing was measured (Fig. 3). The degree of semisolid aspiration was measured using the penetration-aspiration scale (PAS).

All the VFSS procedures were recorded with a camcorder that could run at 30 frames per second. All the images were saved in a personal computer and were analyzed using a multimedia player (GOMplayer, Gretech, Korea). In addition, measurements of laryngeal elevation, post-swallow pharyngeal remnant, and UES width were performed with a 2D screen AutoCAD (Autodesk, San Rafael, CA, USA). As the patient's forward and backward movements and left and right movements during the VFSS might affect the measurements of the remnant and width, the size of the image was readjusted based on the maximum length of a 100 -won coin (23 $\mathrm{mm}$ in diameter) attached to the neck of a patient to minimize the error.

\section{Statistical analysis}

The five factors that were measured in this study were calculated using mean \pm standard deviation. The ShapiroWilk test was used to determine the distributions of these five continuous variables. Each variable showed a normal distribution ( $p>0.05$ by the Shapiro-Wilk test), and we used the parametric statistical analysis. In a $2 \times 2$ crossover design, the analysis of the carry-over effect was based on the sum of two observations for each sequence, and the analysis of the period effect was based on the mean difference. Independent t-test was performed as appropriate to analyze the carry-over and period effects. There were no significant carry-over and period effects in each variable ( $p>0.05$ by the independent $t$-test). Therefore, paired t-test was used to investigate the association between capping of the tracheostomy tube and the 5 VFSS variables. All statistical analyses were performed with SPSS ver. 18.0 (SPSS Inc., Chicago, IL, USA). The pvalues less than 0.05 were considered statistically significant.

\section{RESULTS}

The mean age of the patients was $43.8 \pm 17.2$ years. There were 16 males (53.33\%) and 14 females (46.66\%). The cause of the stroke was hemorrhage in 15 patients $(50.00 \%)$ and ischemia in 15 patients $(50.00 \%)$. With respect to the site of the lesion, it was cortex+subcortex in 9 patients $(30.00 \%)$, cortex in 4 patients $(13.33 \%)$, subcortex in 9 patients $(30.00 \%)$, and brain stem in 8 patients $(26.66 \%)$. With respect to the side of paralysis, it was left hemiplegia in 7 patients $(23.33 \%)$, right hemiplegia in 11 patients (36.66\%), and quadriplegia in 12 patients $(40 \%)$. The mean time from onset to VFSS was $286.7 \pm 71.3$ days, and the mean duration of the tracheostomy was $270.4 \pm 71.7$ days. The mean score of the Korean-Mini Mental State Examination (K-MMSE) was $4.7 \pm 6.0$ points. The mean score of the functional dysphagia scale was $43.3 \pm 14.0$ points. With respect to the type of feeding, it was nasogastric tube in 17 patients (56.66\%) and percutaneous endoscopic gastrostomy tube in 13 patients $(43.33 \%)$ (Table 1$)$.
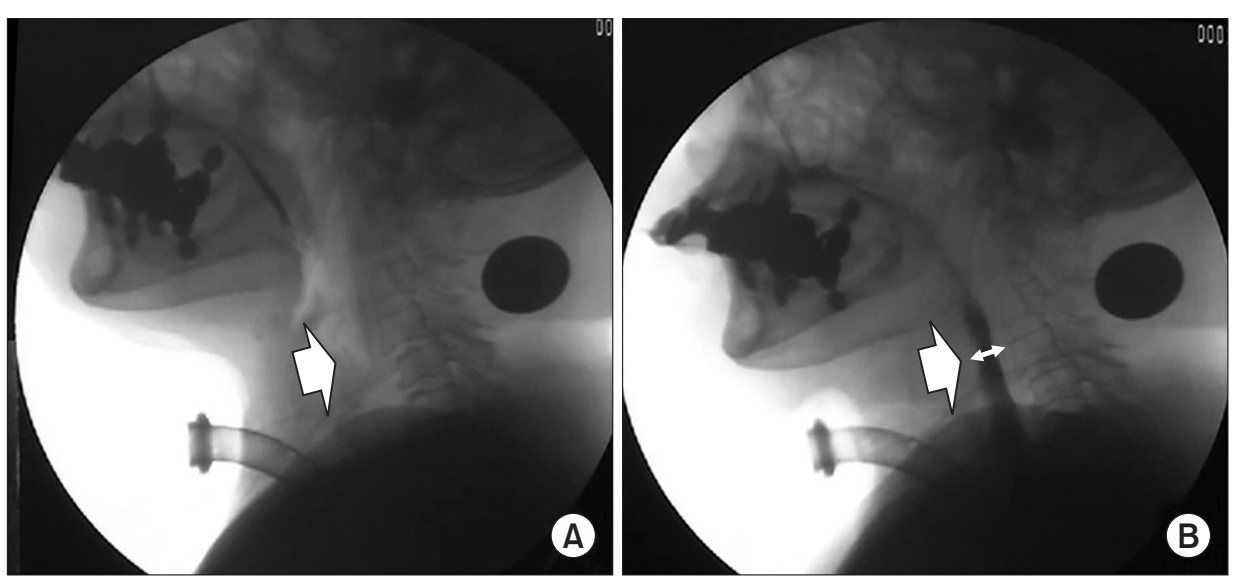

Fig. 3. Upper esophageal sphincter width: the maximal opening of the narrowest region of C3-6 measured with an AutoCAD 2D screen was measured in terms of the anteroposterior diameter $(\mathrm{mm})$. (A) Before swallowing. (2) During swallowing. 
In the results of assessment of differences in swallowing parameters during swallowing with capping and without capping, statistically significant differences were found in the post-swallow pharyngeal remnant (without capping, $48.19 \% \pm 28.70 \%$; with capping, $25.09 \% \pm 19.23 \%$; $\mathrm{p}<0.001$ ), normalized residue ratio scale for valleculae (NRRSv) (without capping, $0.17 \pm 0.12$; with capping, $0.09 \pm$ $0.12 ; \mathrm{p}=0.013)$, normalized residue ratio scale for piriform sinus (NRRSp) (without capping, $0.16 \pm 0.12$; with capping, 0.10 $\pm 0.07 ; \mathrm{p}=0.015$ ), and UES width (without capping, $3.32 \pm 1.61 \mathrm{~mm}$; with capping, $4.61 \pm 1.95 \mathrm{~mm}$; $\mathrm{p}=0.003$ ). However, there were no statistically significant differences in laryngeal elevation ( $\mathrm{x}$-axis without capping, $2.48 \pm 1.45 \mathrm{~mm}$; with capping, $3.26 \pm 2.37 \mathrm{~mm}$; $\mathrm{y}$-axis without capping, $11.11 \pm 5.24 \mathrm{~mm}$; with capping, $12.64 \pm 6.16 \mathrm{~mm}$ ), pharyngeal transit time (without capping, $9.19 \pm 10.14 \mathrm{~s}$; with capping, $9.09 \pm 10.21 \mathrm{~s}$ ), and semisolid aspiration (PAS score) (without capping, 4.94 \pm 2.83 ; with capping, 4.18 \pm 2.24 ) (Table 2).

\section{DISCUSSION}

With tracheostomy tube capping, a decrease in the post-swallow pharyngeal remnant and an increase in the UES width were observed in the VFSS performed in stroke patients who received tracheostomy.

The deglutitive subglottic air pressure generated in the pharyngeal phase of swallowing was lowered in tracheostomy patients because despite the adduction of the true vocal folds, airflow escapes underneath it during swallowing $[14,19]$. As it was confirmed that there was
Table 1. Baseline characteristics of the subjects

\begin{tabular}{|lc}
\hline \multicolumn{1}{|c}{ Characteristic } & Value \\
\hline Age (yr) & $43.8 \pm 17.2$ \\
\hline Gender & \\
\hline Male & $16(53.33)$ \\
\hline Female & $14(46.66)$ \\
\hline Cause of stroke & $15(50.00)$ \\
\hline Hemorrhage & $15(50.00)$ \\
\hline Ischemia & \\
\hline Site of the lesion & $9(30.00)$ \\
\hline Cortex+subcortex & $4(13.33)$ \\
\hline Cortex & $9(30.00)$ \\
\hline Subcortex & $8(26.66)$ \\
\hline Brain stem & $7(23.33)$ \\
\hline Types of impairment & $11(36.66)$ \\
\hline Lt. hemiplegia & $12(40.00)$ \\
\hline Rt. hemiplegia & $286.7 \pm 71.3$ \\
\hline Quadriplegia & $270.4 \pm 71.7$ \\
\hline Time from onset to VFSS (day) & $4.7 \pm 6.0$ \\
\hline Duration of tracheostomy (day) & $43.3 \pm 14.0$ \\
\hline K-MMSE & $17(56.66)$ \\
\hline FDS & $13(43.33)$ \\
\hline Type of feeding & \\
\hline Nasogastric tube & \\
\hline PEG & \\
\hline
\end{tabular}

Values are presented as mean \pm standard deviation or number (\%).

VFSS, videofluoroscopic swallowing study; K-MMSE, Korean-Mini Mental State Examination; FDS, functional dysphagia scale; PEG, percutaneous endoscopic gastrostomy.

Table 2. Comparison of the VFSS measurements before and after capping of the tracheostomy tube

\begin{tabular}{|lccc}
\hline \multicolumn{1}{c}{ Variable } & Without capping & With capping & p-value \\
\hline Laryngeal elevation x-axis (mm) & $2.48 \pm 1.45$ & $3.26 \pm 2.37$ & 0.524 \\
\hline Laryngeal elevation y-axis (mm) & $11.11 \pm 5.24$ & $12.64 \pm 6.16$ & 0.494 \\
\hline Pharyngeal transit time (s) & $9.19 \pm 10.14$ & $9.09 \pm 10.21$ & 0.335 \\
Semisolid aspiration (PAS score) & $4.94 \pm 2.83$ & $4.18 \pm 2.24$ & 0.214 \\
Post-swallow pharyngeal remnant (\%) & $48.19 \pm 28.70$ & $25.09 \pm 19.23$ & $<0.001^{*}$ \\
NRRSv & $0.17 \pm 0.12$ & $0.09 \pm 0.12$ & $0.013^{*}$ \\
NRRSp & $0.16 \pm 0.12$ & $0.10 \pm 0.07$ & $0.015^{*}$ \\
\hline UES width (mm) & $3.32 \pm 1.61$ & $4.61 \pm 1.95$ & $0.003^{*}$ \\
\hline
\end{tabular}

Values are presented as a mean \pm standard deviation.

VFSS, videofluoroscopic swallowing study; PAS, penetration-aspiration scale; NRRSv, normalized residue ratio scale for the valleculae; NRRSp, normalized residue ratio scale for the piriform sinus; UES, upper esophageal sphincter. ${ }^{*} \mathrm{p}<0.05$ by paired $\mathrm{t}$-test. 
a laryngeal subglottic mechanoreceptor [22], which is known to be associated with swallowing motor control [16], sufficient deglutitive subglottic air pressure acts as an important component in the swallowing function. Thus, drastic reduction of the pressure may hinder the laryngeal mechanoreceptor drive. This may, in turn, reduce the bolus transit amount and accordingly lead to reduced UES width, decrease in transit time, and decrease in laryngeal elevation [19]. Therefore, preservation of the deglutitive subglottic air pressure can act as a key factor in dysphagia alleviation. To date, various attempts have been made to alleviate dysphagia by increasing the deglutitive subglottic air pressure. For example, a one-way speaking valve creates subglottic pressure based on the principle that it opens at the time of inhalation and closes at the time of exhalation. By attaching the valve to a cuffless or deflated tracheostomy tube, swallowing can be improved and aspiration can be reduced $[17,18]$, and the positive subglottic air pressure can be restored through decannulation [15].

Previous studies utilized various methods for preserving the subglottic pressure, but many outcomes are controversial when it comes to deriving a clear conclusion on the effect of tube capping in tracheostomy patients. A study showed reduced aspiration in the environment of tube closure [19]. On the other hand, when occlusion of the tracheostomy tube was attempted in 11 of 20 patients (after excluding 9 patients who failed to meet the inclusion criteria; Table 1) who received tracheostomy, occlusion of the tracheostomy tube showed no significant effect on the prevalence of aspiration [23]. There are various reasons for occurrence of aspiration in dysphagia. Besides epiglottic dysmotility and post-swallow remnant, aspiration has been observed in patients with vocal cord palsy (vocal folds cannot close adequately to protect the airway during swallowing) [24], in cases when relaxation and contraction of the upper esophageal sphincter are not achieved sufficiently during swallowing, and in patients with cricopharyngeal dysfunction [25]. Previous studies did not control the factor that can affect aspiration. As a result, it is considered to have shown a different result. In addition, there was lack of consistency even in the characteristics of patients. However in the studies mentioned above, the patients who had a history of stroke were excluded, and the subjects had a limited ability to tolerate tracheostomy tube occlusion during VFSS.
On the contrary, this study targeted only stroke patients. This study measured the factors, including those in previous studies compared to non-enforcement, such as aspiration, laryngeal elevation, pharyngeal transit time, postswallow remnant and UES width. As a result, reduction in the post-swallow pharyngeal remnant and an increase in the UES width were observed.

Post-swallow pharyngeal remnant is caused by incomplete pharyngeal peristalsis. It appears as a result of an increase in the resistance due to UES opening abnormality, cricopharyngeal dysfunction, and impaired pharyngeal propulsion. It may increase the risk of post-swallow overflow aspiration depending on the residue amount [26]. Different ways of improving the pharyngeal remnant include balloon dilatation across the UES [27], swallowing of a balloon of a Foley catheter [28], botulinum toxin injection into the cricopharyngeal muscle $[29,30]$, etc. In this study, post-swallow pharyngeal remnants were reduced, which can be explained to have occurred as a result of an increase in the compelling force in the bolus transport due to the increase in the subglottic pressure that occurred during swallowing.

During safe and normal swallowing without aspiration, it is necessary that the UES opens rapidly and in a timely manner in the pharyngeal phase. Impaired UES relaxation, decreased UES compliance, weak hyoid movement, and inefficient pharyngeal transition may cause abnormality of UES opening [31]. The duration of UES opening is dependent on the amount of swallowed bolus $[32,33]$. Thus, increased UES opening, which was shown in this study, could be associated with reduced postswallow remnants.

In this study, improvement in laryngeal elevation and pharyngeal transit time before and after capping of the tracheostomy tube was not observed. Laryngeal elevation before and after capping did not show a significant difference, and the pharyngeal transit time is thought to reflect the same result. With respect to its mechanism, there was no change in laryngeal elevation because of the anchoring of the larynx to the anterior neck through the tracheostomy [34]. In this study, during swallowing with capping, improvement of semisolid aspiration was not observed, which is consistent with the findings of previous studies [23,35]. However, this study was limited to a single swallow, and repetitive swallows with capping could lessen post-swallow pharyngeal remnants com- 
pared to repetitive swallows without capping. This means that it could increase the swallowing efficiency. Multiple swallows is also one of the factors that affect clearing of remnant. Thus, more studies assessing the long-term effects of swallowing with capping are required with respect to the association with the presence of aspiration, post-swallow pharyngeal remnant, and UES width. In addition, as aspiration acts not only through the pharyngeal remnant but also through complex factors, if studies targeting only a homogenous group are performed, from which patients with vocal cord palsy and cricopharyngeal dysfunction are excluded, more consistent outcomes may be obtained.

The major limitations of this study are as follows: The effects of capping of the tracheostomy tube in each subgroup that was divided according to the dysphagia mechanism were not analyzed. Thus, further studies should be performed for reflecting this point. The other limitations were the relatively small number of subjects and the lack of long-term follow-up. Also, there was no direct measurement of the subglottic pressure. During swallowing, analysis of the subglottic air pressure can be performed through percutaneous puncture of the cricothyroid membrane [14]. Further investigation is needed to determine if there is a direct correlation between the deglutitive subglottic air pressure and tracheostomy tube occlusion.

After tracheostomy tube capping was attempted in stroke patients who underwent tracheostomy, a reduction in post-swallow pharyngeal remnants and an increase in the UES width were observed. Capping of the tracheostomy tube is significant as it can cause dysphagia alleviation in patients who show remnants in the VFSS among stroke patients who have received a tracheostomy tube. Further studies need to be conducted on the direct measurement of the deglutitive subglottic air pressure, double and multiple swallows, and the long-term effects of tracheostomy tube capping on swallowing.

\section{CONFLICT OF INTEREST}

No potential conflict of interest relevant to this article was reported.

\section{REFERENCES}

1. Goldenberg D, Ari EG, Golz A, Danino J, Netzer A, Joachims HZ. Tracheotomy complications: a retrospective study of 1130 cases. Otolaryngol Head Neck Surg 2000;123:495-500.

2. Das P, Zhu H, Shah RK, Roberson DW, Berry J, Skinner ML. Tracheotomy-related catastrophic events: results of a national survey. Laryngoscope 2012;122:30-7.

3. Mann G, Hankey GJ, Cameron D. Swallowing disorders following acute stroke: prevalence and diagnostic accuracy. Cerebrovasc Dis 2000;10:380-6.

4. Falsetti P, Acciai C, Palilla R, Bosi M, Carpinteri F, Zingarelli A, et al. Oropharyngeal dysphagia after stroke: incidence, diagnosis, and clinical predictors in patients admitted to a neurorehabilitation unit. J Stroke Cerebrovasc Dis 2009;18:329-35.

5. Martino R, Foley N, Bhogal S, Diamant N, Speechley M, Teasell R. Dysphagia after stroke: incidence, diagnosis, and pulmonary complications. Stroke 2005;36:2756-63.

6. Bosel J. Tracheostomy in stroke patients. Curr Treat Options Neurol 2014;16:274.

7. Walcott BP, Kamel H, Castro B, Kimberly WT, Sheth $\mathrm{KN}$. Tracheostomy after severe ischemic stroke: a population-based study. J Stroke Cerebrovasc Dis 2014; 23:1024-9.

8. Rabinstein AA, Wijdicks EF. Outcome of survivors of acute stroke who require prolonged ventilatory assistance and tracheostomy. Cerebrovasc Dis 2004; 18:325-31.

9. Muz J, Mathog RH, Nelson R, Jones LA Jr. Aspiration in patients with head and neck cancer and tracheostomy. Am J Otolaryngol 1989;10:282-6.

10. DeVita MA, Spierer-Rundback L. Swallowing disorders in patients with prolonged orotracheal intubation or tracheostomy tubes. Crit Care Med 1990;18:132830.

11. Bonanno PC. Swallowing dysfunction after tracheostomy. Ann Surg 1971;174:29-33.

12. Sasaki CT, Suzuki M, Horiuchi M, Kirchner JA. The effect of tracheostomy on the laryngeal closure reflex. Laryngoscope 1977;87(9 Pt 1):1428-33.

13. Leder SB, Ross DA. Incidence of vocal fold immobility in patients with dysphagia. Dysphagia 2005;20:163-7; discussion 168-9. 
14. Gross RD, Steinhauer KM, Zajac DJ, Weissler MC. Direct measurement of subglottic air pressure while swallowing. Laryngoscope 2006;116:753-61.

15. Kim YK, Choi JH, Yoon JG, Lee JW, Cho SS. Improved dysphagia after decannulation of tracheostomy in patients with brain injuries. Ann Rehabil Med 2015;39: 778-85.

16. Gross RD, Mahlmann J, Grayhack JP. Physiologic effects of open and closed tracheostomy tubes on the pharyngeal swallow. Ann Otol Rhinol Laryngol 2003; 112:143-52.

17. Elpern EH, Borkgren Okonek M, Bacon M, Gerstung C, Skrzynski M. Effect of the Passy-Muir tracheostomy speaking valve on pulmonary aspiration in adults. Heart Lung 2000;29:287-93.

18. Stachler RJ, Hamlet SL, Choi J, Fleming S. Scintigraphic quantification of aspiration reduction with the Passy-Muir valve. Laryngoscope 1996;106(2 Pt 1):2314.

19. Eibling DE, Gross RD. Subglottic air pressure: a key component of swallowing efficiency. Ann Otol Rhinol Laryngol 1996;105:253-8.

20. Rumbak MJ, Graves AE, Scott MP, Sporn GK, Walsh FW, Anderson WM, et al. Tracheostomy tube occlusion protocol predicts significant tracheal obstruction to air flow in patients requiring prolonged mechanical ventilation. Crit Care Med 1997;25:413-7.

21. Pearson WG Jr, Molfenter SM, Smith ZM, Steele CM. Image-based measurement of post-swallow residue: the normalized residue ratio scale. Dysphagia 2013;28: 167-77.

22. Adzaku FK. The morphological and functional characteristics of the innervation of the subglottic mucosa of the larynx. Ann R Coll Surg Engl 1980;62:426-31.

23. Leder SB, Tarro JM, Burrell MI. Effect of occlusion of a tracheotomy tube on aspiration. Dysphagia 1996;11: 254-8.

24. Heitmiller RF, Tseng E, Jones B. Prevalence of aspiration and laryngeal penetration in patients with unilateral vocal fold motion impairment. Dysphagia 2000; 15:184-7.

25. Alfonsi E, Merlo IM, Ponzio M, Montomoli C, Tassorelli C, Biancardi C, et al. An electrophysiological approach to the diagnosis of neurogenic dysphagia: implications for botulinum toxin treatment. J Neurol Neurosurg Psychiatry 2010;81:54-60.

26. Eisenhuber E, Schima W, Schober E, Pokieser P, Stadler A, Scharitzer M, et al. Videofluoroscopic assessment of patients with dysphagia: pharyngeal retention is a predictive factor for aspiration. AJR Am J Roentgenol 2002;178:393-8.

27. Kim JC, Kim JS, Jung JH, Kim YK. The effect of balloon dilatation through Video-Fluoroscopic Swallowing Study (VFSS) in stroke patients with cricopharyngeal dysfunction. J Korean Acad Rehabil Med 2011;35:23-6.

28. Kim YK, Choi SS, Choi JH, Yoon JG. Effectiveness of rehabilitative balloon swallowing treatment on upper esophageal sphincter relaxation and pharyngeal motility for neurogenic dysphagia. Ann Rehabil Med 2015;39:524-34.

29. Haapaniemi JJ, Laurikainen EA, Pulkkinen J, Marttila RJ. Botulinum toxin in the treatment of cricopharyngeal dysphagia. Dysphagia 2001;16:171-5.

30. Ahsan SF, Meleca RJ, Dworkin JP. Botulinum toxin injection of the cricopharyngeus muscle for the treatment of dysphagia. Otolaryngol Head Neck Surg 2000; 122:691-5.

31. Dodds WJ, Logemann JA, Stewart ET. Radiologic assessment of abnormal oral and pharyngeal phases of swallowing. AJR Am J Roentgenol 1990;154:965-74.

32. Cook IJ, Dodds WJ, Dantas RO, Massey B, Kern MK, Lang IM, et al. Opening mechanisms of the human upper esophageal sphincter. Am J Physiol 1989;257(5 Pt 1):G748-59.

33. Logemann JA, Pauloski BR, Rademaker AW, Colangelo LA, Kahrilas PJ, Smith CH. Temporal and biomechanical characteristics of oropharyngeal swallow in younger and older men. J Speech Lang Hear Res 2000; 43:1264-74.

34. Kang JY, Choi KH, Yun GJ, Kim MY, Ryu JS. Does removal of tracheostomy affect dysphagia? A kinematic analysis. Dysphagia 2012;27:498-503.

35. Leder SB, Joe JK, Hill SE, Traube M. Effect of tracheotomy tube occlusion on upper esophageal sphincter and pharyngeal pressures in aspirating and nonaspirating patients. Dysphagia 2001;16:79-82. 